\title{
COMPARISON OF MOLECULAR AND SEROLOGICAL TESTS FOR SEVERE ACUTE RESPIRATORY SYNDROME CORONAVIRUS 2 (SARS-COV-2) IN POST-EXPOSURE EMPLOYEES OF THE NEPHROLOGY DEPARTMENT
}

10.36740/WLek202012103

\author{
Marlena Kwiatkowska, Inga Chomicka, Jolanta Malyszko \\ NEPHROLOGY, DIALYSIS AND INTERNAL MEDICINE, WARSAW MEDICAL UNIVERSITY, WARSAW, POLAND
}

\begin{abstract}
Introduction: A novel coronavirus SARS-CoV-2 RNA, detected by reverse-transcription polymerase chain reaction (RT-PCR) was identified as the cause of a cluster of pneumonia cases in Wuhan, China. It rapidly spread, at first in China, then resulting in an epidemic in other countries throughout the world. One of such controversial topics is the issue of diagnostics and interpretation of test for COVID-19. According to Polish and global guidelines, the basis for diagnosis is molecular testing - real-time reverse transcriptasepolymerase chain reaction (RT-PCR). Taking all these data into consideration, the aim of the study was to compare RT-PCR with serological test in our employees post-exposure. According to Polish and global guidelines, the basis for diagnosis is molecular testing, real-time reverse transcriptase-polymerase chain reaction (RT-PCR).

The aim: To compare RT-PCR with serological test in our employees post-exposure.

Material and methods: 79 employees of the Clinic, 19 men and 60 women in the age range 27-69 years were evaluated. Tests were begun four days after information about the positive test in our "Employee 0 " and lasted for 7 days. At first, we made RT-PCR tests on the specimen from nasopharyngeal swab. Then, we accomplished rapid antibodies tests. This test is based on the qualitative assessment of the presence of $\mathrm{IgM}$ and $\mathrm{lg} \mathrm{G}$ antibodies by immunochromatography using a sample of capillary blood from the fingertip. Results: All the tests were negative. No employee developed symptoms during the 7-day follow-up after the end of the tests.

Conclusions: As routine tests for patients have been implemented widely, but similar solutions for employees have not gained popularity. Use of personal protective equipment (PPE) e.g. facemask and shields, transparent screens, disposable medical uniforms, minimalization the contact time, increasing distance from both colleagues and patients (if possible), and strictly follow sanitary procedures largely contributed to the absence of illness in the surveyed group of employees.
\end{abstract}

KEY WORDS: COVID-19, diagnostic test, RT-PCR, healthcare professionals

Wiad Lek. 2020;73(12 p. I):2572-2575

\section{INTRODUCTION}

For several months, the whole world has been struggling with the problem of rapidly spreading infection with a new type of coronavirus called severe acute respiratory syndrome coronavirus 2 (SARS-CoV-2). This virus causes coronavirus disease 2019 (COVID 2019), which is usually benign. It is estimated that up to $50-75 \%$ of individuals with a positive RT-PCR test remain asymptomatic. However, the disease can be severe and even life-threatening in about $10 \%$ of symptomatic patients [1]. The first case was diagnosed in December 2019 in the Chinese city of Wuhan, the capital of Hubei province, Then the disease quickly appeared in other countries, leading to the WHO announcing a pandemic in March 2020. During this time, scientists and doctors from around the world are working to develop methods to deal with this new type of virus. Still, many doubts and questions remain.

One of such controversial topics is the issue of diagnostics and interpretation of test for COVID-19. According to Polish and global guidelines, the basis for diagnosis is molecular testing - real-time reverse transcriptase-polymerase chain reaction (RT-PCR) [2]. This method is not perfect and its sensitivity depends on how the sample was taken. In a study with 205 participants with confirmed coronavirus infection, it was estimated that the highest sensitivity was obtained by collecting bronchoalveolar lavage (93\%). Further, sensitivity is estimated successively: sputum (72\%), nasal swab (63\%) and pharyngeal swab (32\%) [3]. It should also be remembered that the virus can be detected by RT-PCR on the first day of symptomatic infection, however, the highest detectability is within the first week. After about 3 weeks from the onset of symptoms, the positive tests become negative again [4].

Despite this knowledge, there are false positive and false negative results of RT-PCR, which has serious clinical consequences. The reasons for the erroneous results include incorrect material collection, contamination or too early testing in relation to the occurrence of symptoms. In addition, there is more and more information about cases of infected people who, after two negative RT-PCR tests 
Table 1. Distribution of respondents depending on the type of work performed, gender and age.

\begin{tabular}{cccccc}
\hline \multirow{2}{*}{ Position } & \multicolumn{2}{c}{ Sex } & \multicolumn{3}{c}{ Age } \\
\cline { 2 - 6 } & female & male & $<\mathbf{4 0}$ y.o. & $\mathbf{4 1 - 6 0} \mathbf{y . 0}$ & $>\mathbf{6 0}$ \\
\hline Doctor (physician) & 12 & 9 & 4 & 15 & 2 \\
\hline Nurse & 37 & 3 & 10 & 28 & 2 \\
\hline Warden & 4 & 5 & 2 & 7 & - \\
\hline Secretary & 3 & - & 1 & 2 & - \\
\hline Intern & 3 & - & 2 & 1 & - \\
\hline Physiotherapist & - & 1 & - & 2 & - \\
\hline Medical career & 1 & 1 & - & 56 & 4 \\
\hline Total & 60 & 19 & 19 & & \\
\hline
\end{tabular}

(which is a condition for establishing recovery), another test had positive. In our Department we encountered one such case in which it cannot be clearly stated whether the ambiguous results of RT-PCR resulted from incorrect performance of the test, difficulties in its interpretation due to concomitant diseases or reactivation of the virus [5]. Then, several such cases were identified.

The second diagnostic option is serological tests, whose availability is increasing, but they also have some limitations. These tests rely on detection of IgM and IgG antibodies against SARS-COV-2 virus. There are currently over a dozen different antibody cassette tests available from China, Korea and the United States. They seem to be an ideal screening test because they are fast (the result is obtained after about 10-20min), minimally invasive and their sensitivity seems to vary between 64.7-100\% [6]. IgM and IgG antibodies appear in the body 6-15 days after beginning of infection. On average, seroconversion occurs on days 11, 12 and 14 from the onset of symptoms. For this reason, cassette tests detect antibodies with high sensitivity and specificity only after about two weeks of illness [7]. On the other hand, the issue of persisting IgG antibody levels in serum is questionable and requires further research. Recent data indicate that after 5 weeks from the onset of the disease, IgM levels begin to decline to become almost undetectable after 7 weeks [4]. For these reasons, the role of antibody cassette tests in the diagnosis of COVID-19 is debatable. However, the search for possibilities of other applications of serological tests is ongoing.

Comparing the above described diagnostic methods is very difficult and it seems that each of them may have different applications, especially among health care workers. In our Department, SARS COV-2 has been detected in recent days among one of the employees. This person had no clinical symptoms and it was not known where and when the contact with the infected person took place. The ward was closed for epidemiological reasons and we performed a series of serological and molecular studies among staff.

\section{THE AIM}

The aim of the study was to compare RT-PCR with serological test in our employees post-exposure.

\section{MATERIAL AND METHODS}

\section{MATERIAL}

We qualified 79 employees of the Clinic in total for the tests -19 men and 60 women in the age range 27-69 years. Among them, there were 21 doctors, 3 interns, 40 nurses, 9 wardens, 3 secretaries, 2 medical careers, and 1 physiotherapist. The middle personnel is normally divided into two locations (dialysis station and conservative department) and constitutes two separate teams. However, due to the space occupied on one floor and mutual doctors team in both locations, it was justifiable in the current epidemiological situation, to treat everyone as one group. Details of each group are presented in the Table 1 .

The division into age groups is indicative and based on individual experience and common knowledge about the increased incidence of comorbidities with age [8]. Adding this to the higher risk of disease associated with work in health care, especially in hospitals, we have a special risk group. This based on two key factors - individual factors associated with comorbidities and the higher risk of exposure [9].

\section{METHODS}

Tests were begun four days after information about the positive test in our „Employee 0 ” and lasted for 7 days. The date of the test depended on the employee's availability. In the beginning, we made RT-PCR tests. The sample for this test is material taken during a nasopharyngeal swab. The collection technique is particularly important here - according to the information from the laboratory, the swab should not be less than half the distance from the ear to the entrance to the nose and should last at least 10 seconds.

Three days after the start of the tests, we were allowed to accomplish rapid antibodies tests. This test is based on the qualitative assessment of the presence of $\operatorname{IgM}$ and IgG antibodies by immunochromatography. In this case, we needed for testing a sample of capillary blood from the fingertip. Venous whole blood or plasma can also be used for the test, but we don't use this for practical reasons. The collection procedure is the same as for home glucose measurement (for a glucometer). We used disposable, sterile needles 


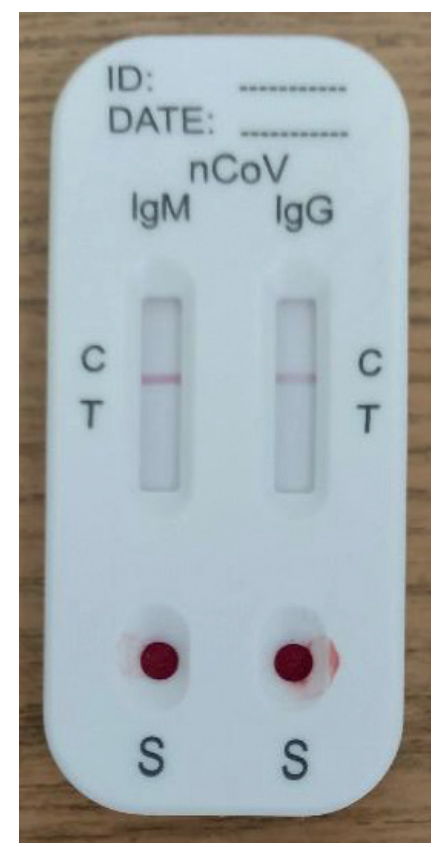

Fig. 1. Rapid antibodies test cassette test (from the Clinic's own collection).

and collected two drops of blood from each subject - one for each type of antibody. After applying the blood to the test window and adding a dedicated solvent, the result is readable after $15 \mathrm{~min}$. Tests performed incorrectly - without the presence of a band in the $\mathrm{C}$ field - were repeated. An example of a correct performer test is shown in Fig. 1.

\section{RESULTS AND DISCUSSION}

From 79 employees, 73 had both tests - RT - PCR and rapid IgM/IgG antibodies (Fig. 2). We started the cassette tests 4 days after the genetic testing and continued for 6 days. Three people had both tests on the same day, two - rapid tests before RT PCR. Regardless of the method or day of collection, all tests were negative.
SARS-CoV-2 RNA is detected by reverse-transcription polymerase chain reaction (RT-PCR) [1]. Flisiak et al. [2] reported that the diagnostics for the COVID-19 infection is discussed, with RT PCR being the basis for the diagnosis of active SARS-CoV-2 infection. Then role of serological methods is briefly presented in this paper, but extensively discussed by Tomasik et al. [6] In the course of an epidemic, mass serological testing with rapid tests "on request", especially for detecting IgM class antibodies, can be used to identify asymptomatic infections once other means of reducing the epidemic have been exhausted. Detection of IgG or IgM/IgG antibodies can be useful in epidemiological studies as suggested by Flisiak et al. [2]. With these tests it is possible to estimate the number of people who have been in contact with the virus and developed antibodies and for population studies. Despite rapid antibody tests are simple, easy to use, fast, cheap, they have important limitations as reported previously [6]. They missed the infection in the early and even mid-phase. They yielded a substantial number of false-negative results, as shown in some countries including Poland [10]. Moreover, to definitively rule out or confirm SARS-CoV-2 infection, the test must be performed with the use of RT PCR molecular diagnostics. Rapid molecular tests recently registered by FDA may offer a possibility for fast track of diagnosis of SARS-CoV-2 infection in emergency departments. It should be stressed that typically RT-PCR of nasopharyngeal swabs has been used to confirm the clinical diagnosis of SARS-CoV-2 infection.

Healthcare workers have an increased risk of exposure to SARS-CoV-2 and in consequence - getting sick or be an asymptomatic transmitter of infection. As we well know the transmission of the virus is dependent on proximity and duration of contact, and the disease itself is also associated with a person's individual risk factors as comorbidities.

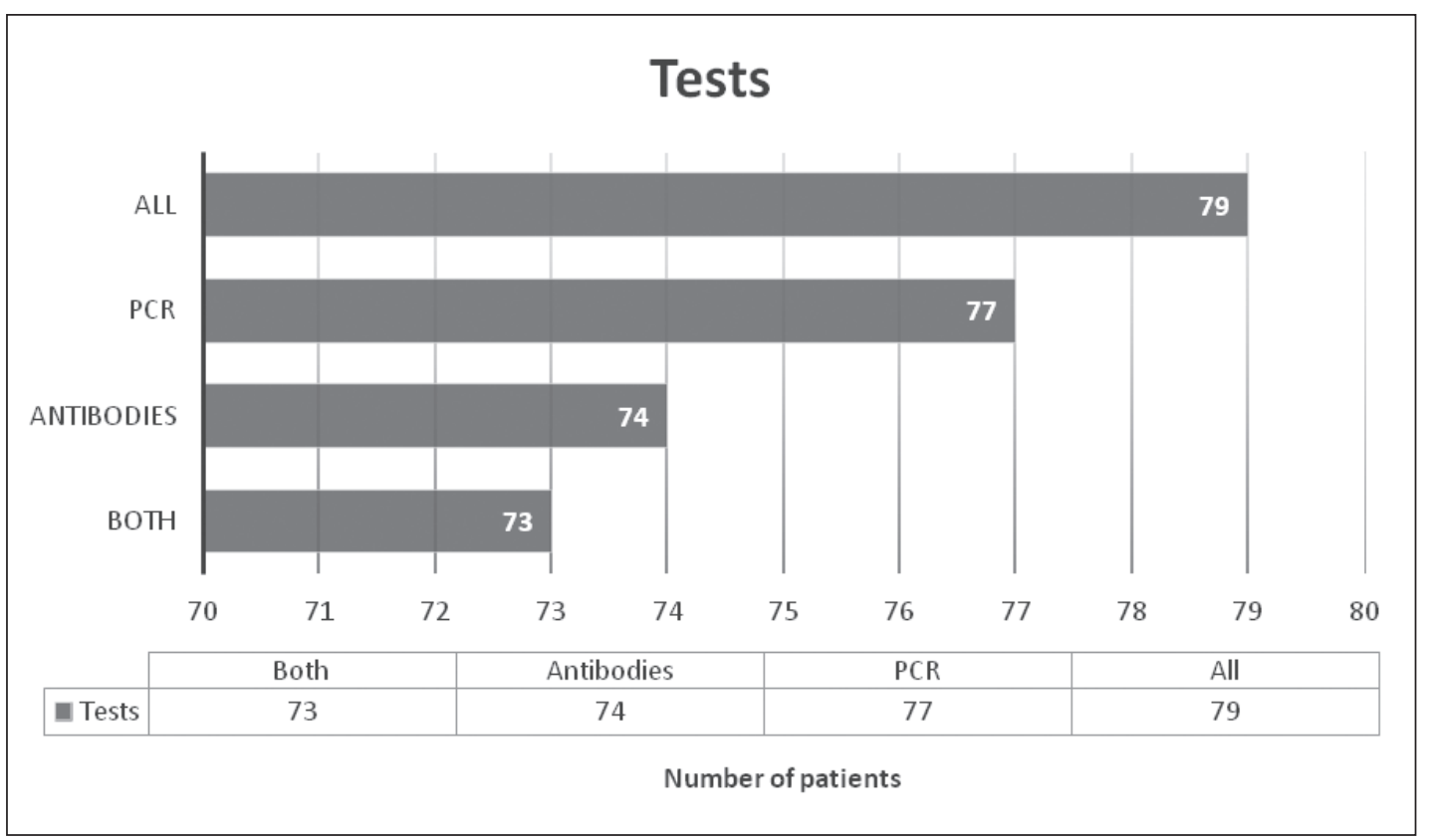

Fig. 2. Summary of the performed tests. 
A team working with a large number of dialysis patients and/or elderly patients with multiple medical burdens is at particularly high risk. Currently, routine tests for patients have been implemented in many places, but similar solutions for employees have not gained popularity. However, taking into account the research sample conducted in our department, one should consider how justified it is to change this position.

Taking into account the reports on the effectiveness of the use of personal protective equipment (PPE) e.g. facemask and shields, transparent screens, disposable medical uniforms, it can be assumed that this is the main key to success [10-12]. In addition to physical barriers, it is also important to reduce the time of contact, increasing distance from both colleagues and patients (if possible), and strictly follow sanitary procedures. In our opinion, these procedures largely contributed to the absence of illness in the surveyed group of employees.

\section{CONCLUSIONS}

1. Negative results should not exclude our vigilance - work environments may have different exposure to the very important factor - aerosol generated during sneezing, coughing or during diagnostic procedures i.e. bronchoscopy.

2. The human factor can also be important.

Therefore, our main task remains a regular and individual risk assessment in a given place and continuous improvement of both: the method of protection and quick response in the event of suspicion of an infection outbreak.

\section{REFERENCES}

1. Pascarella G, Strumia A, Piliego C, et al. COVID-19 diagnosis and management: a comprehensive review. J Intern Med. 2020 Apr 29:10.1111/joim. 13091.

2. Flisiak R, Horban A, Jaroszewicz J, Kozielewicz D, Pawłowska M, et al. Management of SARS-CoV-2 infection: recommendations of the Polish Association of Epidemiologists and Infectiologists as of March 31, 2020. Pol Arch Intern Med. 2020;130(4):352-357.

3. Wang $X, X u Y, G a 0 R$, et al. Detection of SARS-CoV-2 in different types of clinical specimens. JAMA. 2020;323(18):1843-1844.

4. Nandini S, Sundararaj SJ, Akihide R. Interpreting Diagnostic Tests for SARS-CoV-2. JAMA 2020;323(22):2249-2251.

5. Niewiński G, Małyszko J, Niemczyk L, et al. Diagnosis and recovery from severe acute respiratory syndrome coronavirus 2 infection is challenging in kidney patients: tests are an issue. Pol Arch Intern Med. 2020;130:463-465.
6. Tomasik P, Krótki F, Jońca M, Anyszek T. Rapid point-of-care antibody cassette tests for severe acute respiratory syndrome coronavirus 2 : practical consideration. Pol Arch Intern Med. 2020;130:459-462.

7. Coronavirus disease 2019 (COVID-19) in the EU/EEA and the UK - ninth update, Stockholm: ECDC; 2020

8. Docherty $A B$, Harrison EM, Green CA, etal. Features of 20133 UK patients in hospital with covid-19 using the ISARICWHO Clinical Characterisation Protocol: prospective observational cohort study. British Medical Journal 2020;369: m1985.

9. CookTM, Risk to health from COVID-19 for anaesthetists and intensivists - a narrative review. Anaesthesia 2020; doi:10.1111/anae.15220

10. [https://www.washingtonexaminercom/news/coronavirus-testsspain-purchased-from-china-fail-to-detect-positive-cases. Acessed 25.10.2020]

11. Rivett L, SridharS, Sparkes D, et al. Screening of healthcare workers for SARS-CoV-2 highlights the role of asymptomatic carriage in COVID-19 transmission. elife. 2020 May 11;9:e58728

12. Canova V, Lederer Schläpfer H, Piso RJ, et al. Transmission risk of SARSCoV-2 to healthcare workers - observational results of a primary care hospital contact tracing. Swiss Med Wkly. 2020 Apr 25;150:w20257.

\section{ORCID and contributionship}

Marlena Kwiatkowska: 0000-0001-6861-7507 ${ }^{A-F}$

Jolanta Malyszko: 0000-0001-8701-8171 A,C-F

Inga Chomicka: 0000-0001-7472-2266 A-F

\section{Conflict of interest:}

The Authors declare no conflict of interest.

\section{CORRESPONDING AUTHOR Jolanta Malyszko \\ Nephrology, Dialysis and Internal Medicine, Warsaw Medical University, ul. Banacha 1 a, 02-097, Warszawa, Poland tel: +48225992658 e-mail: jolmal@poczta.onet.pl}

Received: 20.10 .2020

Accepted: 03.12.2020

\footnotetext{
A - Work concept and design, B - Data collection and analysis, $\mathbf{C}$ - Responsibility for statistical analysis, D-Writing the article, $\mathbf{E}$-Critical review, $\mathbf{F}$-Final approval of the article
} 